Article Type: Point Counterpoint

\title{
POINT/COUNTERPOINT
}

Suggestions for topics suitable for these Point/Counterpoint debates should be addressed to Jing Cai, The Hong Kong Polytechnic University, Hong Kong: jing.cai@polyu.edu.hk, and/or Habib Zaidi, Geneva University Hospital, Geneva, Switzerland: habib.zaidi@hcuge.ch, and/or Gerald White, Colorado Associates in Medical Phys, Colorado Springs, CO, United States: gerald.white@mindspring.com.Persons participating in Point/Counterpoint discussions are selected for their knowledge and communicative skill. Their positions for or against a proposition may or may not reflect their personal opinions or the positions of their employers.

\section{FLASH Radiotherapy: Newsflash or Flash in The Pan?}

Peter G Maxim, Ph.D.

Department of Radiation Oncology, Indiana University School of Medicine, Indianapolis, IN 46202

(Tel: 317-944-1185; E-mail: pmaxim@iu.edu)

Paul Keall, Ph.D.

ACRF Image X Institute, University of Sydney, Camperdown, NSW, 2006

(Tel: 61-2-8627-1159; E-mail: paul.keall@sydney.edu.au)

Jing Cai, Ph.D., Moderator

This is the author's manuscript of the article published in final edited form as:

Maxim, P. G., Keall, P., \& Cai, J. (2019). FLASH Radiotherapy: Newsflash or Flash in The Pan? Medical Physics, 0(ja). https://doi.org/10.1002/mp.13685 


\section{OVERVIEW}

Ionizing radiation with ultra-high dose rates $(>40 \mathrm{~Gy} / \mathrm{s})$, known as FLASH, has drawn great attention since its introduction in $2014^{1}$. It has been shown to markedly reduce radiation toxicity to normal healthy tissues while inhibiting tumor growth with similar efficiency as compared to conventional dose rate irradiation in pre-clinical models. Some believe that FLASH irradiation holds great promises and is perhaps the biggest finding in recent radiotherapy history. However, others remain skeptical about the replication of FLASH efficacy in cancer patients with concerns about technical complexity, lack of understanding of its molecular radiobiological underpinnings, and reliability. This is the premise debated in this month's Point/Counterpoint.

Arguing for the Proposition is Peter G. Maxim, PhD. Dr. Maxim received his Dipl. Phys. (M.Sc.) in Applied Physics from the Technical University, Berlin, Germany and his Dr. rer. nat. $(\mathrm{PhD})$ in Biophysics from Humboldt University, Berlin, Germany. He started his professional career in medical physics as a postdoctoral fellow at Stanford University where he was subsequently appointed as Assistant Professor. He is certified by the American Board of Radiology in Therapeutic Radiologic Physics and is currently an Associate Professor, Director and Vice Chair of Radiation Oncology Physics at Indiana University. His personal areas of emphasis are stereotactic ablative radiation therapy (SABR), four-dimensional computed tomography (4D CT), and the development of novel, and safe radiation therapy that addresses the major technical challenge of accurately and precisely treating moving targets and anatomy. Peter is interested in the management of interdisciplinary, science- and technology-oriented teams in health care and is actively developing next-generation radiotherapy technologies.

Arguing against the propositions is Paul Keall, PhD. Dr. Keall is a National Health \& Medical Research Council Senior Principal Research Fellow and Director of the ACRF Image X Institute at the University of Sydney. His team of scientists and clinical partners develop and apply novel cancer imaging and targeted radiotherapy methods. One of Dr. Keall's research interests is motion management in radiotherapy: he chaired the TG264, "Safe Clinical Implementation of MLC tracking." and is currently chairing the update to TG76: "The management of respiratory motion in radiation oncology (TG324)". Dr. Keall 
has published over 300 papers and has 18 awarded patents. He is a Fellow of AAPM and sits on the Editorial Board of Medical Physics.

\section{FOR THE PROPOSITION: Peter G Maxim, Ph.D.}

\section{Opening Statement}

Ultra-high dose rate irradiation (in sub-second time scales) or "FLASH" (the term coined by Favaudon et al. in $2014^{1}$ ), has recently gained attention because of the surprising observation of markedly increased therapeutic index compared to conventional dose rate irradiation (in minutes) in preclinical studies of in vivo animal models. In fact, normal tissue sparing by FLASH has been described at least as far back as 1966 by Hornsey and Alper ${ }^{2}$, who attributed this finding to depletion of oxygen in tissues at very high dose rates ${ }^{3}$, but has been relatively dormant until the recent renaissance of interest.

The increased therapeutic index of FLASH compared to conventional dose rate irradiation, or the "FLASH effect," has now been reported in multiple preclinical models. Normal tissue sparing by FLASH of multiple organ systems including lung, brain, intestinal tract, and skin has been demonstrated in multiple mouse strains and even additional species (cat and mini-pig), while demonstrating an equivalent (and in some cases superior) tumoricidal effect relative to conventional dose-rate delivery in multiple in vivo tumor models ${ }^{1,4-8}$. Given the nascent state of the field, a large portion of experimental observations to date remain preliminary and unpublished, and many questions remain unanswered particularly with respect to mechanism. While there are data suggesting a fundamental physical-chemical effect, i.e., radiochemical depletion of oxygen at FLASH dose rates, modulation of inflammatory cytokines, e.g., TGF- $\beta$, and differential immunologic responses between tumor and normal tissues, these could be downstream effects rather than independent mechanisms. Certainly, we have barely scratched the surface of potential mechanistic pathways.

The FLASH effect is also incompletely characterized even from a phenomenological standpoint. Evidence to date suggests that a dose rate threshold of approximately 40 Gray/second or higher is needed to produce the FLASH effect ${ }^{1,6}$. But the accelerator-based radiation delivery systems used for FLASH experiments to date produce pulsed radiation, and 
there are many aspects of delivery speed, e.g., total dose and delivery time, dose per pulse, pulse timing structure, etc., that may be critical to the effect and have not been comprehensively evaluated, which poses an experimentally daunting combinatorial problem.

To date, preclinical FLASH irradiation of small animals and small, superficial targets in larger animals has been possible using modifications of existing irradiation systems that are capable of producing FLASH dose rates when limited to small volumes (of up to a few cubic centimeters), including electron linear accelerators ${ }^{9,10}$, a synchrotron light source producing kilovoltage energy x-rays ${ }^{5}$, and certain proton accelerators ${ }^{11}$.

All together, these data provide strong evidence that the observed FLASH effect across multiple species and organ systems is real, which makes this dramatic finding the biggest 'Newsflash' in recent radiotherapy history.

\section{AgAinST THE PROPOSITION: Paul Keall, Ph.D.}

\section{Opening Statement}

FLASH therapy is an interesting concept that is attracting scientists, clinicians and industry. With the sobering findings of the Global Task Force on Radiotherapy for Cancer $\mathrm{Control}^{12}$, demonstrating the staggering worldwide need for radiation therapy, any technology that can reduce treatment times and enable more patients to be treated per machine is admirable. Faster treatments will also change the dose distribution to the lymphocytes in the circulating blood, potentially impacting the patient's immune response. Understanding the biological mechanisms underpinning the effectiveness of FLASH therapy may provide new insights into current radiobiological theories. However, before jumping on the FLASH bandwagon, we should ask some questions:

1. Does FLASH delivery technology exist for humans? No. And it will not exist for the foreseeable future. Ignoring VMAT, let's assume that a state-of-the-art treatment would require 5-7 intensity modulated beams. As massive amounts of radiation are being delivered in a short time, real-time adaptive radiotherapy would be necessary to

This article is protected by copyright. All rights reserved. 
ensure the delivered dose is close to the planned dose. To achieve the benefits of FLASH and delivering the entire dose quickly, the following technologies - that currently don't exist - need to be developed:

a. A configuration of 5-7 FLASH beams to be delivered simultaneously.

b. Fast FLASH intensity modulation. Currently limited by leaf speed, intensity modulation on a sub-second scale requires new technology to be built.

c. Real-time FLASH adaptation. Given the higher requirements on motion management for stereotactic radiation therapy, ${ }^{13}$ it is even more important for the FLASH beam to be adapted in real-time to ensure the beam and the target are aligned.

2. Will FLASH be cost effective? No. Given 5-7 beam lines are required, either 5-7 beam generators are needed, or technology analogous to electron CT would have to be developed. The Task Force ${ }^{12}$ concluded that radiation therapy needs are highest in low and middle income countries. Therefore, FLASH is incompatible with global needs.

3. Will treatment times be reduced with FLASH therapy? Possibly. However, the impact may be small. Current high dose treatments with dual-arc VMAT can be delivered in two minutes of a 15-minute time slot. The remainder of the time in the room is patient set-up, immobilization and pre-treatment imaging. Developments in real-time adaptive radiation therapy may reduce the need for complex immobilization and pretreatment imaging. Reducing the $>80 \%$ of the time the beam is not on, rather than the $<20 \%$ of the time the beam is on, will have a larger impact.

4. Are the controls questionable in FLASH experiments? Sometimes. Creating FLASH beams that match conventional beams is challenging. It is difficult to change just one variable, the dose rate. For example, in one of the first in vivo FLASH studies, ${ }^{14}$ mice with lung tumors were treated with Cs-137 photons in the conventional arm, and with 4.5 MeV electrons in the FLASH arm. The FLASH arm showed lower lung toxicity. However, is this difference explained by physics or biology? Cs-137 photons deliver higher doses outside of the tumor, whereas $4.5 \mathrm{MeV}$ electrons have lower surface dose

This article is protected by copyright. All rights reserved. 
and higher central dose in mice. In another study, the results of square conventional beams were compared with FLASH circular beams, albeit of similar equivalent field size. ${ }^{15}$ Every FLASH study should be carefully reviewed to evaluate the impact of these differences, in addition to dose rate, on the results.

In summary, FLASH is an interesting concept that has caught the imagination of many people. Am I on the FLASH bandwagon? No. I remain an interested spectator.

\section{Rebuttal: Peter G Maxim, Ph.D.}

I appreciate Dr. Keall's insights and valid arguments in his opening statement.

Dr. Keall stated that FLASH RT is currently not available for humans, and it will not exist in the foreseeable future either. It is true, as of today, FLASH has only been studied in preclinical settings and there are no published studies reporting on clinical translation. Will it not exist in the foreseeable future? I disagree.

Recent advances in linear accelerator science and radiofrequency power generation and distribution technologies have led to prototypes of compact, high-efficiency linear accelerators suitable for producing high-energy x-rays (in the megavoltage energy range) or very high-energy electrons (exceeding $100 \mathrm{MeV}$ energy) capable of treating large-volume, deep-seated targets at FLASH dose rates. In particular, investigators at Stanford University (Billy W Loo, Jr., MD, PhD), SLAC National Accelerator Laboratory (Sami G Tantawi, $\mathrm{PhD}$ ), and Indiana University (Peter G Maxim, $\mathrm{PhD}$ ), are engaged in a program to develop a medical linear accelerator system based on these technologies, called pluridirectional highenergy agile scanning electronic radiotherapy (PHASER), designed to deliver highly conformal FLASH intensity-modulated radiation therapy to general radiation therapy targets ${ }^{16}$.

The core enabling and technical innovations of PHASER-based FLASH include: (1) highly compact, economical, and power-efficient linear accelerator and radiofrequency (RF) power sources based on fundamentally new accelerator science able to produce hundred-fold greater beam output than conventional medical linacs; (2) a rapid RF power-distribution system that supplies an array of 16 stationary beamlines providing a full complement of noncoplanar (conical geometry) beams for highly conformal radiotherapy without mechanical gantry rotation; (3) rapid electronically scanned highly intensity-modulated photon beams 
from scanning an electron beam on a stationary target and collimator array, eliminating the need for mechanical collimator motion; and (4) integration of full ring diagnosticquality/speed multi-detector CT, sharing a common isocenter with the non-coplanar treatment beamlines. Besides providing position verification immediately before and during rapid treatment delivery, fast diagnostic-quality CT facilitates an efficient adaptive planning work flow in which a scan at the beginning of a treatment session can be used for plan adaptation, for example by rapid selection from a set of pre-calculated plan permutations or fully reoptimizing, depending on time and computational tradeoffs ${ }^{17}$. Such a workflow would allow higher patient throughput and reduces treatment room utilization times.

With respect to the cost-effectiveness of PHASER, compactness, power efficiency, and economical manufacturing are key design considerations and we anticipate the overall cost to be like that of conventional linacs, which makes PHASER the ideal technology for globally accessible curative cancer care.

The motivation for developing this technology was to provide a fundamental solution to mitigating uncertainty due to organ motion, by combining rapid volumetric image-guidance and treatment delivery that could be completed faster than the time scale of physiologic motion. The same delivery speed would enable clinical evaluation of FLASH biological effects.

In conclusion, FLASH promises to be a paradigm shift in curative radiation therapy with preclinical evidence of fundamentally improved therapeutic index. If this remarkable finding is translatable to humans, the switch to the PHASER technology will become mandatory.

This article is protected by copyright. All rights reserved. 


\section{Rebuttal: Paul Keall, Ph.D.}

My esteemed colleague has given a concise summary of the FLASH literature. When interpreting this literature, we should scientifically assess the FLASH results. For example, one of the articles referenced includes the claim that FLASH sparing has been demonstrated in cat and mini-pig species, the studies closest to human applications. ${ }^{18} \mathrm{We}$ find that for the cat treatments, this was a single arm study, demonstrating only that radiation therapy works. In the mini-pig treatment, the FLASH vs. conventional results were positive. However, there was only one subject. The $95 \%$ confidence intervals on an $n=1$ study span $2.5 \%$ to $100 \%$.

One of the key drivers for FLASH are the biological changes. The studies to date have investigated only early effects of radiation. Even if these early changes are real, late effects may be better or worse with FLASH. As Darby has shown us with breast treatment, ${ }^{19}$ serious late effects continue more than 30 years after radiation therapy. What are the late effects with FLASH? Also, FLASH works against one of the four R's of radiobiology, reoxygenation. Hypoxia is one of the biggest negative prognostic indicators of cancer treatment outcome. ${ }^{20}$ Acute hypoxia varies on a timescale of minutes. ${ }^{21}$ With FLASH therapy, there is no time for changes in acute hypoxia to occur during treatment. Conventional therapy enables the delivery to be more biologically effective.

With an analogy to another emerging technology, early radiomics studies were performed without independent test sets. Multiple hypotheses were tested without appropriate Bonferroni correction. There are now clear guidelines for radiomics studies. ${ }^{22}$ FLASH studies could benefit from such guidelines. To convince a scientist - and scientists should be convinced by data alone - a forward pathway for FLASH research and development is to:

1. Perform controlled FLASH vs. conventional studies with the dose rate as the single controlled variable,

2. Understand the radiobiology underpinning FLASH,

3. Verify scientific findings independently,

4. Appreciate the experimental limitations and quantify their estimated impact on the results,

5. Solve, or define viable solutions for, all the technical problems necessary for human FLASH therapy listed in point 1 of my opening statement. 
The scientists, authors, reviewers and editors involved with FLASH therapy need to carefully approach the subject and acknowledge the limitations of their studies. Overcoming these limitations will drive innovation. I will watch this space with interest.

Finally, I leave you with the Oxford dictionary definition of FLASH: to shine in a bright but brief way.

I would like to thank Helen Ball, Magdalena Bazalova-Carter and Amit Sawant for excellent and interesting input to this exciting topic.

\section{Conflicts of Interest}

Dr. Maxim is a founder of TibaRay, Inc. Dr. Keall has no relevant conflict of interest.

\section{References}

1. Favaudon V, Caplier L, Monceau V, et al. Ultrahigh dose-rate FLASH irradiation increases the differential response between normal and tumor tissue in mice [published online ahead of print 2014/07/18]. Sci Transl Med. 2014;6(245):245ra293.

2. Hornsey S, Alper T. Unexpected dose-rate effect in the killing of mice by radiation [published online ahead of print 1966/04/09]. Nature. 1966;210(5032):212-213.

3. Hornsey S, Bewley DK. Hypoxia in mouse intestine induced by electron irradiation at high dose-rates [published online ahead of print 1971/01/01]. Int J Radiat Biol Relat Stud Phys Chem Med. 1971;19(5):479-483.

4. Loo BW, Schuler E, Lartey FM, et al. Delivery of Ultra-Rapid Flash Radiation Therapy and Demonstration of Normal Tissue Sparing After Abdominal Irradiation of Mice. Int J Radiat Oncol. 2017;98(2):E16-E16.

5. Montay-Gruel P, Bouchet A, Jaccard M, et al. X-rays can trigger the FLASH effect: Ultra-high dose-rate synchrotron light source prevents normal brain injury after whole brain irradiation in mice [published online ahead of print 2018/09/05]. Radiother Oncol. 2018;129(3):582-588.

6. Montay-Gruel P, Petersson K, Jaccard M, et al. Irradiation in a flash: Unique sparing of memory in mice after whole brain irradiation with dose rates above 100Gy/s [published online ahead of print 2017/05/27]. Radiother Oncol. 2017;124(3):365-369.

7. Schueler E, Trovati S, King G, et al. FLASH Irradiation Improves the Therapeutic Index Following GI Tract Irradiation. Med Phys. 2016;43(6):3783-3783.

8. Vozenin MC, De Fornel P, Petersson K, et al. The Advantage of FLASH Radiotherapy Confirmed in Mini-pig and Cat-cancer Patients [published online ahead of print 2018/06/08]. Clin Cancer Res. 2019;25(1):35-42.

This article is protected by copyright. All rights reserved. 
9. Jaccard M, Duran MT, Petersson K, et al. High dose-per-pulse electron beam dosimetry: Commissioning of the Oriatron eRT6 prototype linear accelerator for preclinical use. Med Phys. 2018;45(2):863-874.

10. Schuler E, Trovati S, King G, et al. Experimental Platform for Ultra-high Dose Rate FLASH Irradiation of Small Animals Using a Clinical Linear Accelerator [published online ahead of print 2016/11/07]. Int J Radiat Oncol Biol Phys. 2017;97(1):195-203.

11. Patriarca A, Fouillade C, Auger M, et al. Experimental Set-up for FLASH Proton Irradiation of Small Animals Using a Clinical System. Int J Radiat Oncol. 2018;102(3):619-626.

12. Atun R, Jaffray DA, Barton MB, et al. Expanding global access to radiotherapy. The Lancet Oncology. 2015;16(10):1153-1186.

13. Benedict SH, Yenice KM, Followill D, et al. Stereotactic body radiation therapy: The report of AAPM Task Group 101. Med Phys. 2010;37(8):4078-4101.

14. Favaudon V, Caplier L, Monceau V, et al. Ultrahigh dose-rate FLASH irradiation increases the differential response between normal and tumor tissue in mice. Science translational medicine. 2014;6(245):245ra293-245ra293.

15. Montay-Gruel P, Bouchet A, Jaccard M, et al. X-rays can trigger the FLASH effect: Ultra-high dose-rate synchrotron light source prevents normal brain injury after whole brain irradiation in mice. Radiotherapy and Oncology. 2018;129(3):582-588.

16. Maxim PG, Tantawi SG, Loo BW. PHASER: A platform for clinical translation of FLASH cancer radiotherapy. Radiotherapy and Oncology. In press. https://doi.org/10.1016/j.radonc.2019.05.005.

17. Cherry Kemmerling EM, Wu M, Yang H, Maxim PG, Loo BW, Jr., Fahrig R. Optimization of an on-board imaging system for extremely rapid radiation therapy [published online ahead of print 2015/11/02]. Med Phys. 2015;42(11):6757-6767.

18. Vozenin M-C, De Fornel P, Petersson K, et al. The advantage of Flash radiotherapy confirmed in mini-pig and cat-cancer patients. Clinical Cancer Research. 2019;25(1):35-42.

19. Darby SC, Ewertz M, McGale P, et al. Risk of ischemic heart disease in women after radiotherapy for breast cancer. New England Journal of Medicine. 2013;368(11):987998.

20. Vaupel P, Mayer A. Hypoxia in cancer: significance and impact on clinical outcome. Cancer and Metastasis Reviews. 2007;26(2):225-239.

21. Kirkpatrick J, Cardenas-Navia L, Dewhirst M. Predicting the effect of temporal variations in PO2 on tumor radiosensitivity. Int J Radiat Oncol Biol Phys. 2004;59(3):822-833.

22. Collins GS, Reitsma JB, Altman DG, Moons KG. Transparent reporting of a multivariable prediction model for individual prognosis or diagnosis (TRIPOD): the TRIPOD statement. BMC medicine. 2015;13(1):1.

This article is protected by copyright. All rights reserved. 

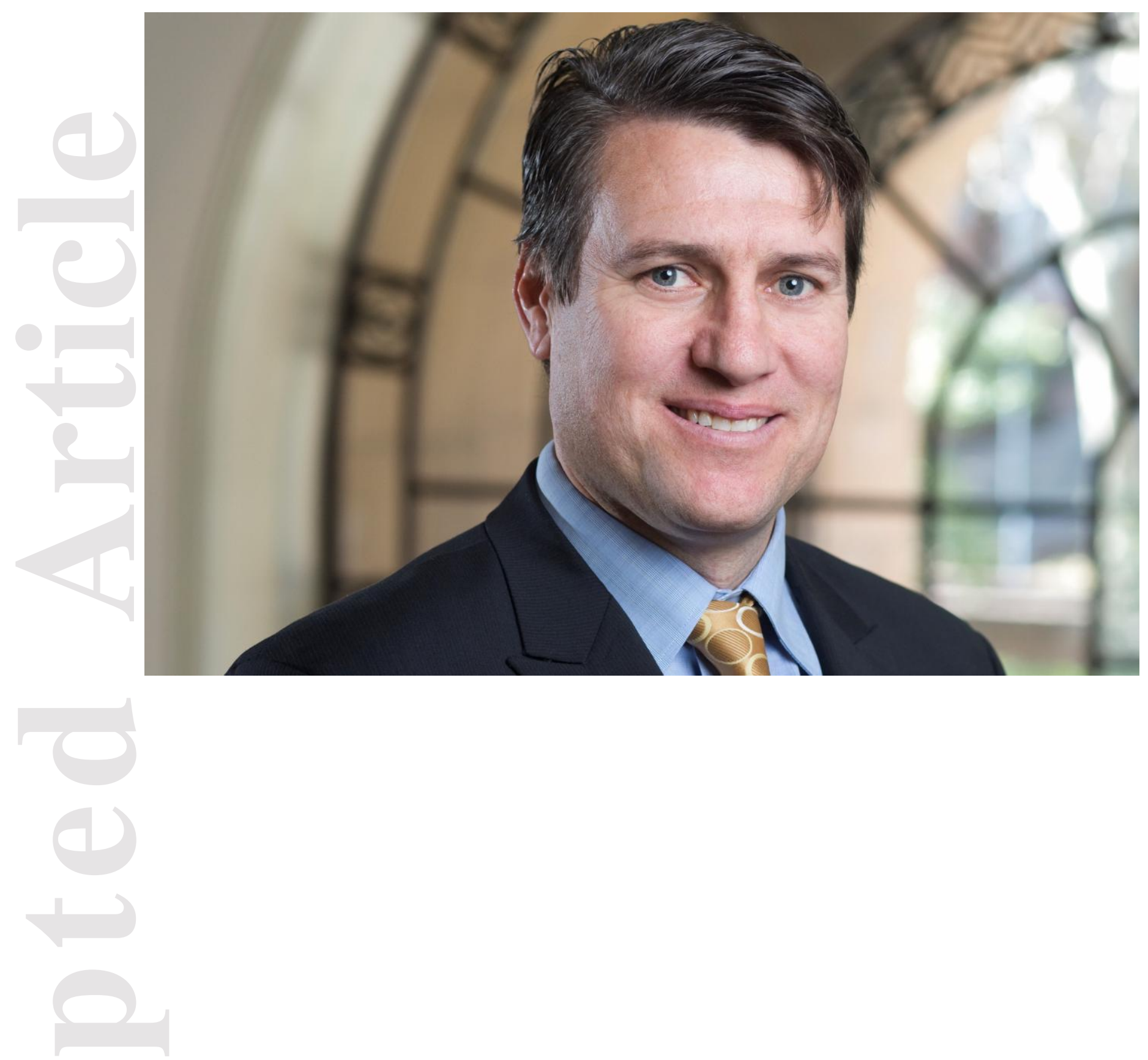

This article is protected by copyright. All rights reserved. 


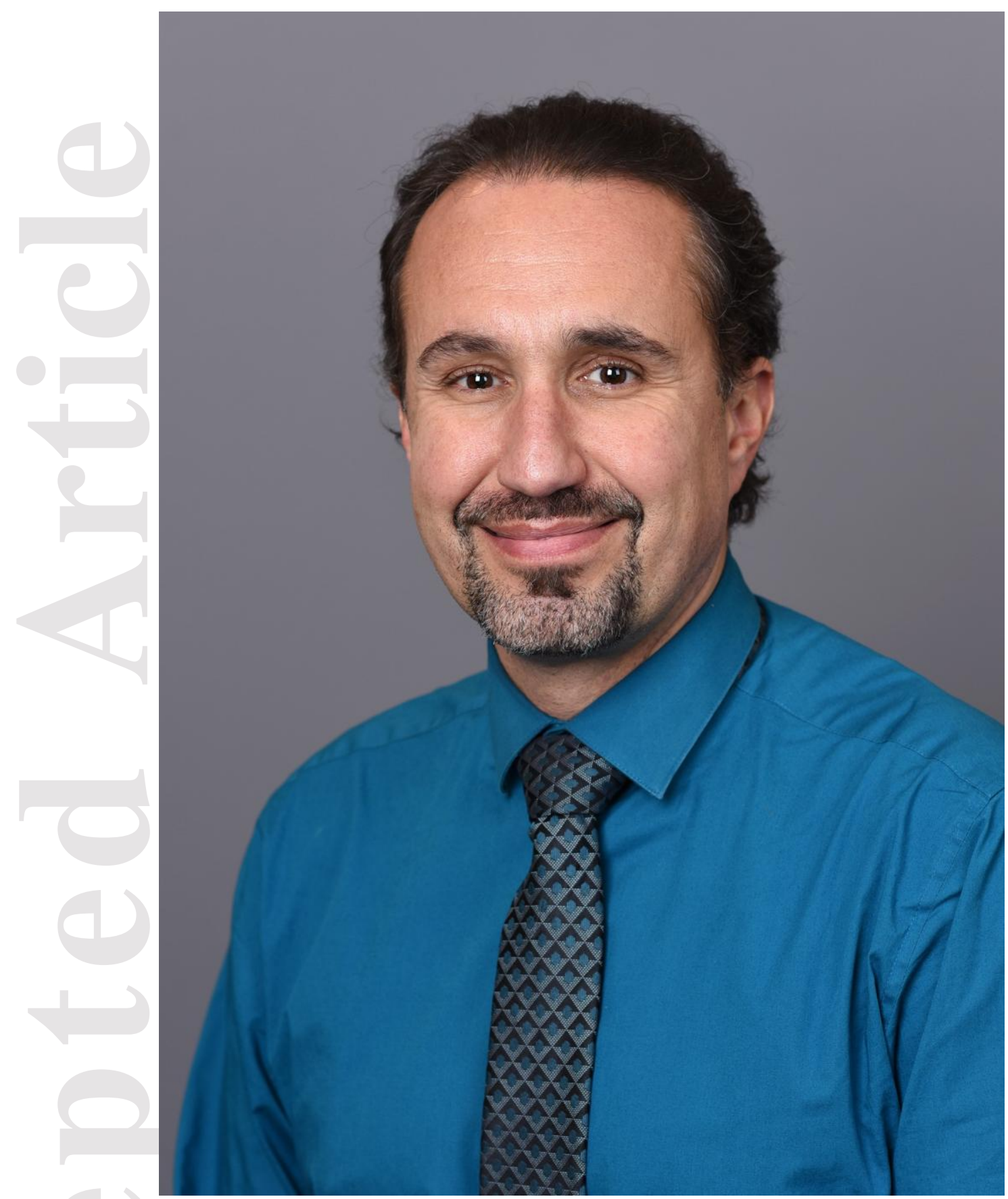

This article is protected by copyright. All rights reserved. 


\section{RAD formation}

\section{Automate Your Treatment Planning}

\section{Workflow in Eclipse TPS.}

\begin{tabular}{|c|c|c|c|c|c|c|c|c|}
\hline \multirow[b]{2}{*}{$\begin{array}{l}\text { Senturo: } \\
\text { lemelse. }\end{array}$} & \multicolumn{8}{|c|}{ Prostate Structure Checks } \\
\hline & $\begin{array}{c}\text { Structure } \\
\text { Mun }\end{array}$ & 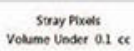 & Holes la Struxture & Sike Guss & Laterably & $\begin{array}{l}\text { Contrasicting } \\
\text { Constraints }\end{array}$ & so Viean & Powifal \\
\hline PTV & PV & $\checkmark$ & $\checkmark$ & $\checkmark$ & & & 30 & $\checkmark$ \\
\hline Femuer. & PEMURREI & $\checkmark$ & $\checkmark$ & $\checkmark$ & $\begin{array}{l}\text { Rion: } \\
\gamma \text { Right }\end{array}$ & & so & $\checkmark$ \\
\hline remuct & renvelu & $\checkmark$ & $\checkmark$ & $\checkmark$ & $\operatorname{lem}_{100}$ & & $\infty$ & $\checkmark$ \\
\hline
\end{tabular}

\section{Prostate Margin Checks}

\begin{tabular}{|c|c|c|c|c|c|c|c|c|c|c|}
\hline $\begin{array}{l}\text { xpandes } \\
\text { itructive } \\
\text { emolste }\end{array}$ & 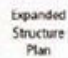 & $\begin{array}{l}\text { Source } \\
\text { Structure } \\
\text { Templiste }\end{array}$ & $\begin{array}{l}\text { souvces } \\
\text { Structure } \\
\text { Plan }\end{array}$ & Not & Pest & reght & Lett & Sup & Int & Prosphat \\
\hline & PTV & CTV & $\mathrm{GN}$ & 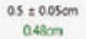 & $\begin{array}{l}0.5+0.050=1 \\
0.770 m\end{array}$ & $\begin{array}{c}0.5+\cos \mathrm{cm} \\
0.05 \mathrm{cos}\end{array}$ & $\begin{array}{c}\text { Cst ocsen } \\
\text { CAABor }\end{array}$ & $\begin{array}{c}0.5+0.050 m \\
0.4500\end{array}$ & $\begin{array}{c}0.5+0.005 \mathrm{~cm} \\
0.77 \mathrm{~cm}\end{array}$ & $\checkmark$ \\
\hline
\end{tabular}

\section{Conventional Plan Checks}

\begin{tabular}{|c|c|c|c|c|}
\hline Fin Check & Epected & Prostater:100 & Pandfal & Comment \\
\hline Thoten Dose Cratre on Aborthem & Aat 13023 & ansosis & $x$ & \\
\hline Phoser Vetune Dose Gro Siet (en) & 0.25 & 0.28 & $\checkmark$ & \\
\hline Phaten Hertero oenenty & ON & or & $\checkmark$ & \\
\hline ef siles thachess (con) & 0.25 & es & X & \\
\hline $\begin{array}{l}\text { Navimum Nunber af CT Sices in } 30 \\
\text { Laspo }\end{array}$ & 5280 & 32 & $\checkmark$ & \\
\hline 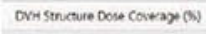 & $2100 \%$ & $2100 \%$ & $\checkmark$ & \\
\hline
\end{tabular}

\section{EZFluence}

\section{Automated 3D Planning}

Automate field in field planning.

Automate skin flash.

Breast, Whole Brain, larynx,

extremities, and more.

$85 \%$ time savings

\section{$\checkmark$ ClearCheck}

\section{One-Click Plan Evaluation}

Check every dose constraint. Automate plan checking. Easily compare treatment plans. One-click printing.

Save over $\$ 33,000$ per planner/yr

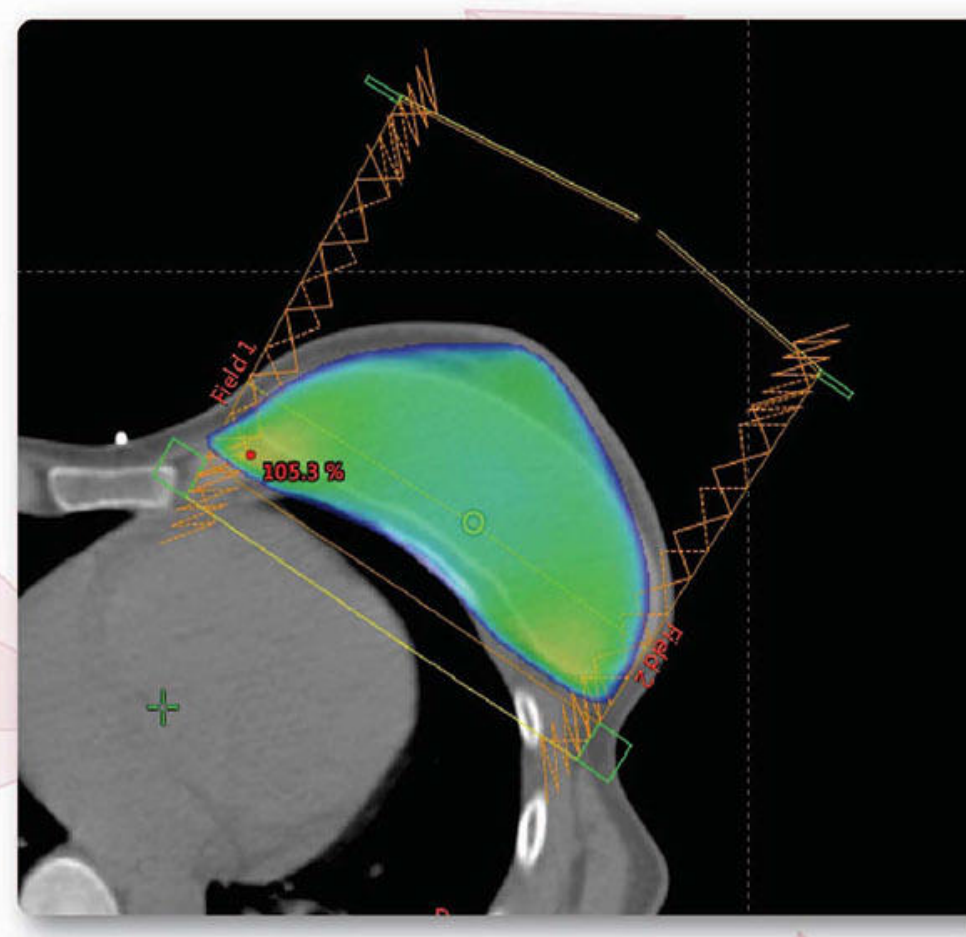

